\title{
A New Hybrid Estimator for the Generalized Weibull Family Distribution
}

\author{
Hesham M. Riad ${ }^{1}$ \& Ahmed M. Hashish ${ }^{1}$ \\ ${ }^{1}$ College of Business \& Economics, Qassim University, Kingdom of Saudi Arabia \\ Correspondence: Hesham M. Riad, College of Business \& Economics, Qassim University, Kingdom of Saudi \\ Arabia. E-mail: hesham_reyad@yahoo.com
}

Received: April 29, 2014 Accepted: July 14, 2014 Online Published: August 4, 2014

doi:10.5539/jmr.v6n3p114

URL: http://dx.doi.org/10.5539/jmr.v6n3p114

\begin{abstract}
The method of moments (MOM) is suffered from a trouble in their corresponding estimators for the bounded distributions, which is nonfeasibility. In the sense that the supports inferred from the estimates fail to contain all observations. In this paper, we introduce a new hybrid estimator based on the MOM estimators for the generalized Weibull family distribution (GWFD). Monte Carlo simulation is performed to compare the hybrid moments estimators with the associated MOM estimators in terms of bias and root mean square error. The proposed hybrid estimator is easy to use, always feasible and it has more desirable properties than the associated MOM estimators.
\end{abstract}

Keywords: auxiliary constraint, feasible estimates, hybrid estimator, method of moments, Monte Carlo simulation, generalized Weibull family distribution

\section{Introduction}

The MOM has a disadvantage in their estimates for the bounded distributions, which is nonfeasibility. These distributions are bounded by their parameters, thus the upper (lower) bound of these distributions is not belonging to real line but depending on their parameters. The MOM for these distributions does not guarantee that their respective estimates will be consistent with the observed data. i.e., one or more of the observed data could be larger (smaller) than the estimated upper (lower) bound and thus MOM estimators would not be feasible. Actually these estimators are not accurate which decreases the advantages of using MOM technique in estimating the unknown parameters for these bounded distributions. Dupuis (1996a, 1996b) calculated the probability of obtaining nonfeasible MOM and probability weighted moments estimates for the generalized Pareto and generalized extreme value distributions respectively. The hybrid moment estimator is a new procedure for estimating bounded distributions suggested by Dupuis and Taso (1998) which incorporates an auxiliary constraint on feasibility into the estimates obtained from the MOM for the generalized Pareto and generalized extreme value distributions to yield feasible estimates. Hassan and Riad (2004) introduced hybrid estimators based on MOM and probability weighted moments estimators for the three parameter Weibull distribution and showed that the hybrid estimators are performed better than the corresponding MOM and probability weighted moments estimators in terms of bias and root mean square error. In this paper, we introduce a hybrid estimator based on the MOM estimators for the generalized Weibull family distribution (GWFD). We investigate the distributional properties of this estimator by using Monte Carlo simulation.

\section{The Generalized Weibull Family}

The generalized Weibull family first suggested in Mudholkar et al. (1991) for constructing isotones, has the following quantile function $Q(u)$, the distribution function $F(x)$ and the probability density function $f(x)$ :

$$
\begin{gathered}
Q(u)= \begin{cases}\sigma\left[1-(1-u)^{\lambda} / \lambda\right]^{\alpha}, & \lambda \neq 0, \\
\sigma[-\log (1-u)]^{\alpha}, & \lambda=0 .\end{cases} \\
F(x)=1-\left[1-\lambda(x / \sigma)^{1 / \alpha}\right]^{1 / \lambda},
\end{gathered}
$$

and 


$$
f(x)=\frac{1}{\alpha \sigma}\left[1-\lambda(x / \sigma)^{1 / \alpha}\right]^{(1 / \lambda)-1}(x / \sigma)^{(1 / \alpha)-1} .
$$

Where $0<x<\frac{\sigma}{\lambda^{\alpha}}, \sigma>0, \alpha>0$ and $-\infty<\lambda<\infty$. While $\sigma$ is the scale parameter and $1 / \alpha, 1 / \lambda$ are two shape parameters. The generalized Weibull family yields the weibull family when $\lambda=0$, the exponential distribution for $\alpha=1, \lambda=0$, and the log-logestic distribution for $\lambda=-1$, which is often used as a model in survival studies. Moreover, common parametric distributions such as the lognormal and the Gama distributions are very well approximated by members of the family; see Mudholkar and Kollia (1994). Further analysis of the generalized Weibull family, including examination of the skewness and Kurtosis, density shapes and tail characteristics, extreme value distributions, density classification, and its relation to the Pearson system and other distributions can be found in Mudholkar and Kollia (1994).

\section{The Moments Estimation}

Let $X_{1}, X_{2}, \ldots, X_{n}$ be i.i.d. random sample from a population whose density function is a generalized Weibull family distribution (GWFD) given in Equation (3). We have the $r^{\text {th }}$ population moments about zero given by

$$
\mu_{r}^{\prime}=E\left(x^{r}\right)=\frac{1}{\alpha \sigma} \int_{0}^{\frac{\sigma}{\alpha^{\alpha}}} x^{r}\left[1-\lambda(x / \sigma)^{1 / \alpha}\right]^{(1 / \lambda)-1}(x / \sigma)^{(1 / \alpha)-1} d x
$$

Substituting by $y=1-\lambda(x / \sigma)^{1 / \alpha}$, so Eq (4) yields

$$
\mu_{r}^{\prime}=\frac{\sigma^{r}}{\lambda^{\alpha r+1}} \quad \beta\left[\frac{1}{\lambda}, \alpha r+1\right]
$$

where $\beta(\cdot, \cdot)$ is the beta function, and the $r^{\text {th }}$ sample moments given by

$$
m_{r}^{\prime}=n^{-1} \sum_{i=1}^{n} x_{i}^{r}
$$

The MOM estimators $\tilde{\alpha}, \tilde{\sigma}$ and $\tilde{\lambda}$ of $\alpha, \sigma$ and $\lambda$ respectively can be obtained by equating Equation (5) by Equation (6) for $r=1,2,3$ and solving the resulting equations for $\tilde{\alpha}, \tilde{\sigma}$ and $\tilde{\lambda}$, thus we have the following system of equations:

$$
\begin{gathered}
\frac{\tilde{\sigma}}{\tilde{\lambda}^{\tilde{\alpha}+1}} \beta\left(\tilde{\alpha}+1, \frac{1}{\tilde{\lambda}}\right)=\bar{x} \\
\frac{\tilde{\sigma}^{2}}{\tilde{\lambda}^{2 \tilde{\alpha}+1}} \beta\left(2 \tilde{\alpha}+1, \frac{1}{\tilde{\lambda}}\right)=\frac{1}{n} \sum_{i=1}^{n} x_{i}^{2} \\
\frac{\tilde{\sigma}^{3}}{\tilde{\lambda}^{3} \tilde{\alpha}+1} \beta\left(3 \tilde{\alpha}+1, \frac{1}{\tilde{\lambda}}\right)=\frac{1}{n} \sum_{i=1}^{n} x_{i}^{3}
\end{gathered}
$$

From Equation (7), we obtain

$$
\tilde{\sigma}=\frac{\tilde{\lambda}^{\tilde{\alpha}+1} \bar{x}}{\beta\left(\tilde{\alpha}+1, \frac{1}{\tilde{\lambda}}\right)}
$$

By substitution with Equation (10) in Equation (8), we can get

$$
\frac{\tilde{\lambda} \bar{x}^{2} \beta\left(2 \tilde{\alpha}+1, \frac{1}{\lambda}\right)}{\beta^{2}\left(\tilde{\alpha}+1, \frac{1}{\lambda}\right)}=\frac{1}{n} \sum_{i=1}^{n} x_{i}^{2}
$$

Subtracting $\bar{x}^{2}$ from both sides of Equation (11), we get

$$
\frac{\tilde{\lambda} \beta\left(2 \tilde{\alpha}+1, \frac{1}{\lambda}\right)}{\beta^{2}\left(\tilde{\alpha}+1, \frac{1}{\lambda}\right)}=\frac{s^{2}}{\bar{x}^{2}}+1
$$

By substitution with Equation (10) in Equation (9), we can obtain

$$
\frac{\tilde{\lambda}^{2} \bar{x}^{3} \beta\left(3 \tilde{\alpha}+1, \frac{1}{\lambda}\right)}{\beta^{3}\left(\tilde{\alpha}+1, \frac{1}{\lambda}\right)}=\frac{1}{n} \sum_{i=1}^{n} x_{i}^{3}
$$


A numerical solution and computer facilities are needed for evaluating Equation (12) and Equation (13) simultaneously to obtain $\tilde{\lambda}, \tilde{\alpha}$ then substitution in Equation (10) to obtain $\tilde{\sigma}$.

\section{The Hybrid Moments Estimation}

The MOM estimators are not feasible when the following auxiliary constraint is violated

$$
x_{(n: n)} \leq \frac{\tilde{\sigma}}{\tilde{\lambda}^{\tilde{\alpha}}}
$$

We introduce hybrid estimator $\left(\sigma^{*}, \lambda^{*}, \alpha^{*}\right)$ based on the MOM estimators which always satisfies Equation (14) given by $\lambda^{*}=\tilde{\lambda}, \alpha^{*}=\tilde{\alpha}$, and

$$
\sigma^{*}= \begin{cases}\tilde{\sigma}, & x_{(n: n)} \leq \frac{\tilde{\sigma}}{\tilde{\lambda}^{\tilde{\alpha}}} \\ \sigma^{\prime \prime}, & \text { otherwise }\end{cases}
$$

where $\sigma^{\prime \prime}=\tilde{\lambda}^{\tilde{\alpha}} x_{(n: n)}$.

The potential usefulness of the hybrid estimators given in Equation (15) which always satisfy Equation (14) solving the problem of obtaining nonfeasible MOM estimators are showed in the following interpretation. These discussion is concerned here to the estimator $\sigma^{*}$ due to the estimators $\lambda^{*}, \alpha^{*}$ are the same of that obtained by the method of MOM. If the auxiliary constraint given in Equation (14) is exist, the hybrid estimator $\sigma^{*}$ is equivalent with the similar of that obtained by the method of MOM, $\tilde{\sigma}$, i.e., the estimated upper bound $\frac{\tilde{\sigma}}{\tilde{\alpha}^{\tilde{\alpha}}}$ being consistent with the observed data, where it cannot find any observation doesn't contained in this estimator which equivalent to be feasible estimator. In the other hand, if Equation (14) doesn't exist, the hybrid estimator $\sigma^{*}$ is differ from of that obtained by the method of MOM, $\tilde{\sigma}$, i.e. the estimated upper bound being inconsistent with the observed data, which means it doesn't contain all observations in this estimator which equivalent to be nonfeasible estimator. In the other hand, if Equation (13) doesn't exist, the hybrid estimator $\sigma^{*}$ is differ from of that obtained by the method $\frac{\tilde{\sigma}}{\tilde{\tilde{\alpha}} \tilde{\alpha}}$ of MOM, $\tilde{\sigma}$, i.e., the estimated upper bound $\frac{\tilde{\sigma}}{\tilde{\alpha} \tilde{\alpha}}$ being inconsistent with the observed data, which means it doesn't contain all observations in this estimator which equivalent to be nonfeasible estimator. But the estimated upper bound obtained by the hybrid estimator is equivalent to

$$
\frac{\sigma^{\prime \prime}}{\tilde{\lambda}^{\tilde{\alpha}}}=\frac{\tilde{\lambda}^{\tilde{\alpha}} x_{(n: n)}}{\tilde{\lambda}^{\tilde{\alpha}}}=x_{(n: n)}
$$

Which indicate that the estimated upper bound is equal to the largest observation, i.e., the the estimated upper bound is transformed to be consistent with the observed data which equivalent to say that it changed to be feasible estimator.

\section{Comparison of Estimators}

Statistical experiments are carried out to compare the hybrid estimator; $\sigma^{*}$ with the original MOM estimator; $\tilde{\sigma}$ in terms of their bias and rmse. Simulation were performed for sample sizes $n=5,15,50,100,200$ with shape parameters take the values; $\lambda=0.7,0.8,0.4,0.1$ and $\alpha=0.2,0.4$. The scale parameter take the values; $\sigma=0.1,0.5,0.8,1,1.2,1.4,1.6,1.8$ and 2. For each combination of values of $n, \lambda, \alpha$ and $\sigma, 1000$ random samples were generated from the generalized Weibull family distribution. The parameters are estimated under two procedures: the MOM and the hybrid based on the MOM. Tables (1-10) shows the bias and rmse for the estimators $\sigma^{*}, \tilde{\sigma}$ and for different values of $\alpha, \sigma$ and $\lambda$. We have three cases:

1) If $\lambda>\alpha$ : It can be seen from Tables $(1,2,3,4)$ if $0.1 \leq \sigma \leq 0.8$ that the MOM and hybrid estimators have the same result in both bias and rmse. If $\sigma=1$, the MOM estimator has smaller bias and rmse than the hybrid estimator, and if $1.2 \leq \sigma \leq 2$, the hybrid estimator is much better than the MOM estimator in both criteria bias and rmse.

2) If $\lambda=\alpha$ : It can deducted from Tables $(5,6)$ if $0.1 \leq \sigma \leq 0.5$ that the performance of the MOM and hybrid estimators is equivalent in both bias and rmse. But for $0.8 \leq \sigma \leq 2$, the MOM estimator has smaller bias and rmse than the hybrid estimator.

3) If $\lambda<\alpha$ : It can be seen from Tables $(7,8,9,10)$ if $0.1 \leq \sigma \leq 0.5$ that the MOM and hybrid estimators have the same performance in both bias and rmse. If $0.8 \leq \sigma \leq 1.4$ the hybrid estimator has greater bias and rmse with the associated MOM estimator, and if $1.6 \leq \sigma \leq 2$, in some cases the hybrid estimator is much better than the MOM estimator when $(\lambda=0.1, \alpha=0.2)$ in tables $(7,8)$, and in other cases the MOM estimator has favorable properties when $(\lambda=0.1, \alpha=0.4)$ in Tables $(9,10)$. 


\section{Conclusion Remarks}

A new hybrid estimator is introduced for estimating the unkown parameters of the (GWFD). The hybrid estimator is built on by adding an auxiliary constraint in the MOM estimators to yield feasible estimators. The hybrid estimator for the (GWFD) much better in many cases with both bias and rmse specially when $\lambda>\alpha$.

\section{References}

Dupuis, D. J. (1996a). Estimating the probability of obtaining non feasible parameter estimates of the generalized Pareto distribution. Journal of Statistical Computation and Simulation, 54, 249-267. http://dx.doi.org/10.1080/00949659608811728

Dupuis, D. J. (1996b). Estimating the probability of obtaining non feasible parameter estimates of the generalized extreme-value distribution. Journal of Statistical Computation and Simulation, 56, 23-38. http://dx.doi.org/10.1080/00949659608811778

Hassan, A. S., \& Riad, H. M. (2004). A comparative study for estimators of the three parameter Weibull distribution. The Annual Conference on Statistics, Computer Science and Operations Research, Institute of Statistical Studies and Research, Cairo University, 39, 25-39.

Mudholkar, G. S., \& Kollia, G. D. (1994). Generalized Weibull family: A structural analysis. Comm.Statist.Theory Meth., 23, 1149-1171. http://dx.doi.org/10.1080/03610929408831309

Mudholkar, G. S., Kollia, G. D., Lin, C. T., \& Patel, K. R. (1991). A graphical procedure for comparing goodnessof-fit tests. JRSS B, 53, 221-232. 


\section{Appendix}

Table 1. Bias amount for estimating $\sigma$ with moment and hybrid moment methods in case of unknown $\lambda, \sigma$ and $\alpha$ for the generalized Weibull distribution (10000 samples are generated with $\lambda=0.7, \alpha=0.2$ and different values of $\sigma$

\begin{tabular}{llccccc}
\hline \multirow{2}{*}{ Method } & \multicolumn{5}{c}{ Sample size } \\
\cline { 3 - 7 } & & 5 & 15 & 50 & 100 & 200 \\
\hline 0.1 & MOM & 0.903 & 0.904 & 0.904 & 0.904 & 0.904 \\
& Hy-MOM & 0.903 & 0.904 & 0.904 & 0.904 & 0.904 \\
\hline 0.5 & MOM & 0.512 & 0.512 & 0.512 & 0.512 & 0.512 \\
& Hy-MOM & 0.512 & 0.512 & 0.512 & 0.512 & 0.512 \\
\hline 0.8 & MOM & 0.217 & 0.218 & 0.218 & 0.218 & 0.218 \\
& Hy-MOM & 0.217 & 0.218 & 0.218 & 0.218 & 0.218 \\
\hline 1 & MOM & 0.021 & 0.021 & 0.022 & 0.002 & 0.022 \\
& Hy-MOM & 0.055 & 0.065 & 0.07 & 0.071 & 0.072 \\
\hline 1.2 & MOM & -0.176 & -0.175 & -0.175 & -0.175 & -0.175 \\
& Hy-MOM & 0.062 & 0.076 & 0.083 & 0.085 & 0.087 \\
\hline \multirow{2}{*}{1.4} & MOM & -0.372 & -0.371 & -0.371 & -0.371 & -0.371 \\
& Hy-MOM & 0.069 & 0.087 & 0.096 & 0.009 & 0.101 \\
\hline 1.6 & MOM & -0.268 & -0.567 & -0.567 & -0.566 & -0.566 \\
& Hy-MOM & 0.074 & 0.097 & 0.109 & 0.113 & 0.115 \\
\hline 1.8 & MOM & -0.764 & -0.763 & -0.762 & -0.762 & -0.762 \\
& Hy-MOM & 0.079 & 0.107 & 0.122 & 0.126 & 0.129 \\
\hline 2 & MOM & -0.959 & -0.958 & -0.0958 & -0.958 & -0.958 \\
& Hy-MOM & 0.083 & 0.117 & 0.134 & 0.14 & 0.143 \\
\hline
\end{tabular}

Table 2. RMSE amount for estimating $\sigma$ with moment and hybrid moment methods in case of unknown $\lambda, \sigma$ and $\alpha$ for the generalized Weibull distribution (10000 samples are generated with $\lambda=0.7, \alpha=0.2$ and different values of $\sigma$ )

\begin{tabular}{llccccc}
\hline \multirow{2}{*}{$\sigma$} & \multirow{2}{*}{ Method } & \multicolumn{5}{c}{ Sample size } \\
\cline { 3 - 7 } & & 5 & 15 & 50 & 100 & 200 \\
\hline 0.1 & MOM & 40.403 & 23.329 & 12.778 & 9.036 & 6.389 \\
& Hy-MOM & 40.403 & 23.329 & 12.778 & 9.036 & 6.389 \\
\hline 0.5 & MOM & 22.88 & 13.219 & 7.242 & 5.121 & 3.621 \\
& Hy-MOM & 22.88 & 13.219 & 7.242 & 5.121 & 3.621 \\
\hline 0.8 & MOM & 9.712 & 5.619 & 3.08 & 2.178 & 1.54 \\
& Hy-MOM & 9.712 & 5.619 & 3.08 & 2.178 & 1.54 \\
\hline 1 & MOM & 0.968 & 0.558 & 0.306 & 0.216 & 0.153 \\
& Hy-MOM & 2.502 & 1.674 & 0.989 & 0.715 & 0.512 \\
\hline 1.2 & MOM & 7.856 & 4.514 & 2.169 & 1.745 & 1.234 \\
& Hy-MOM & 2.882 & 1.972 & 1.177 & 0.854 & 0.613 \\
\hline 1.4 & MOM & 16.627 & 9.576 & 5.242 & 3.706 & 2.62 \\
& Hy-MOM & 3.323 & 2.259 & 1.363 & 0.991 & 0.713 \\
\hline 1.6 & MOM & 25.399 & 14.636 & 8.013 & 8.5 .665 & 4.006 \\
& Hy-MOM & 3.573 & 2.537 & 1.547 & 1.128 & 0.813 \\
\hline 1.8 & MOM & 34.153 & 19.691 & 10.781 & 7.622 & 5.39 \\
& Hy-MOM & 3.872 & 2.806 & 1.728 & 1.264 & 0.912 \\
\hline 2 & MOM & 42.904 & 24.74 & 13.546 & 9.578 & 6.772 \\
& Hy-MOM & 4.17 & 3.076 & 1.907 & 1.398 & 1.011 \\
\hline & & & & & &
\end{tabular}


Table 3. Bias amount for estimating $\sigma$ with moment and hybrid moment methods in case of unknown $\lambda, \sigma$ and $\alpha$ for the generalized Weibull distribution (10000 samples are generated with $\lambda=0.8, \alpha=0.4$ and different values of $\sigma$ )

\begin{tabular}{llccccc}
\hline \multirow{2}{*}{$\sigma$} & \multirow{2}{*}{ Method } & \multicolumn{5}{c}{ Sample size } \\
\cline { 3 - 7 } & & 5 & 15 & 50 & 100 & 200 \\
\hline 0.1 & MOM & 0.909 & 0.909 & 0.909 & 0.909 & 0.909 \\
& Hy-MOM & 0.909 & 0.909 & 0.909 & 0.909 & 0.909 \\
\hline 0.5 & MOM & 0.536 & 0.537 & 0.537 & 0.537 & 0.537 \\
& Hy-MOM & 0.536 & 0.537 & 0.537 & 0.537 & 0.537 \\
\hline 0.8 & MOM & 0.259 & 0.26 & 0.26 & 0.26 & 0.26 \\
& Hy-MOM & 0.259 & 0.26 & 0.26 & 0.26 & 0.26 \\
\hline 1 & MOM & 0.076 & 0.078 & 0.078 & 0.078 & 0.078 \\
& Hy-MOM & 0.078 & 0.081 & 0.086 & 0.088 & 0.641 \\
\hline 1.2 & MOM & -0.105 & -0.103 & -0.102 & -0.102 & -0.102 \\
& Hy-MOM & 0.044 & 0.081 & 0.1 & 0.105 & 0.108 \\
\hline 1.4 & MOM & -0.282 & -0.28 & -0.279 & -0.279 & -0.279 \\
& Hy-MOM & 0.04 & 0.09 & 0.115 & 0.121 & 0.125 \\
\hline 1.6 & MOM & -0.455 & -0.453 & -0.452 & -0.452 & -0.452 \\
& Hy-MOM & 0.033 & 0.097 & 0.129 & 0.137 & 0.142 \\
\hline 1.8 & MOM & -0.622 & -0.62 & -0.62 & -0.62 & -0.62 \\
& Hy-MOM & 0.024 & 0.103 & 0.143 & 0.153 & 0.159 \\
\hline 2 & MOM & -0.779 & -0.778 & -0.779 & -0.779 & -0.779 \\
& Hy-MOM & 0.013 & 0.108 & 0.155 & 0.167 & 0.174 \\
\hline
\end{tabular}

Table 4. RMSE amount for estimating $\sigma$ with moment and hybrid moment methods in case of unknown $\lambda, \sigma$ and $\alpha$ for the generalized Weibull distribution (10000 samples are generated with $\lambda=0.8, \alpha=0.4$ and different values of $\sigma$ )

\begin{tabular}{llccccc}
\hline \multirow{2}{*}{$\sigma$} & \multirow{2}{*}{ Method } & \multicolumn{5}{c}{ Sample size } \\
\cline { 3 - 7 } & & 5 & 15 & 50 & 100 & 200 \\
\hline 0.1 & MOM & 40.62 & 23.464 & 12.853 & 9.088 & 6.426 \\
& Hy- MOM & 40.63 & 23.464 & 12.853 & 9.088 & 6.426 \\
\hline 0.5 & MOM & 23.972 & 13.864 & 7.596 & 5.371 & 3.799 \\
& Hy- MOM & 23.972 & 13.864 & 7.596 & 5.371 & 3.799 \\
\hline 0.8 & MOM & 11.588 & 6.719 & 3.683 & 2.604 & 1.842 \\
& Hy- MOM & 11.588 & 6.719 & 3.683 & 2.604 & 1.842 \\
\hline 1 & MOM & 3.535 & 2.024 & 1.105 & 0.78 & 0.552 \\
& Hy- MOM & 3.535 & 2.103 & 1.214 & 0.886 & 0.641 \\
\hline 1.2 & MOM & 4.868 & 2.673 & 1.449 & 1.024 & 0.723 \\
& Hy- MOM & 3.008 & 2.188 & 1.423 & 1.053 & 0.765 \\
\hline 1.4 & MOM & 12.729 & 7.235 & 3.953 & 2.794 & 1.975 \\
& Hy- MOM & 3.562 & 2.464 & 1.635 & 1.217 & 0.887 \\
\hline 1.6 & MOM & 20.468 & 11.7 & 6.4 & 4.525 & 3.199 \\
& Hy- MOM & 4.271 & 2.725 & 1.84 & 1.377 & 1.007 \\
\hline 1.8 & MOM & 27.952 & 16.018 & 8.767 & 6.2 & 4.383 \\
& Hy- MOM & 5.171 & 2.976 & 2.036 & 1.532 & 1.124 \\
\hline 2 & MOM & 35.04 & 20.116 & 11.016 & 7.791 & 5.508 \\
& Hy- MOM & 6.286 & 3.222 & 2.221 & 1.68 & 1.234 \\
\hline
\end{tabular}


Table 5. Bias amount for estimating $\sigma$ with moment and hybrid moment methods in case of unknown $\lambda, \sigma$ and $\alpha$ for the generalized Weibull distribution (10000 samples are generated with $\lambda=0.4, \alpha=0.4$ and different values of $\sigma$ )

\begin{tabular}{llccccc}
\hline \multirow{2}{*}{$\sigma$} & \multirow{2}{*}{ Method } & \multicolumn{5}{c}{ Sample size } \\
\cline { 2 - 6 } & & 5 & 15 & 50 & 100 & 200 \\
\hline 0.1 & MOM & 0.928 & 0.929 & 0.93 & 0.93 & 0.93 \\
& Hy- MOM & 0.928 & 0.929 & 0.93 & 0.93 & 0.93 \\
\hline 0.5 & MOM & 0.571 & 0.574 & 0.575 & 0.575 & 0.575 \\
& Hy- MOM & 0.571 & 0.574 & 0.575 & 0.575 & 0.575 \\
\hline 0.8 & MOM & 0.301 & 0.305 & 0.306 & 0.306 & 0.306 \\
& Hy- MOM & 0.301 & 0.306 & 0.311 & 0.316 & 0.323 \\
\hline 1 & MOM & 0.123 & 0.127 & 0.128 & 0.128 & 0.128 \\
& Hy- MOM & 0.242 & 0.314 & 0.365 & 0.384 & 0.399 \\
\hline 1.2 & MOM & -0.053 & -0.049 & -0.048 & -0.048 & -0.048 \\
& Hy- MOM & 0.269 & 0.364 & 0.43 & 0.455 & 0.474 \\
\hline 1.4 & MOM & -0.226 & -0.222 & -0.221 & -0.221 & -0.221 \\
& Hy- MOM & 0.292 & 0.411 & 0.394 & 0.525 & 0.548 \\
\hline 1.6 & MOM & -0.395 & -0.391 & -0.39 & -0.39 & -0.39 \\
& Hy- MOM & 0.309 & 0.455 & 0.554 & 0.592 & 0.621 \\
\hline 1.8 & MOM & -0.557 & -0.554 & -0.554 & -0.553 & -0.553 \\
& Hy- MOM & 0.324 & 0.496 & 0.613 & 0.658 & 0.691 \\
\hline 2 & MOM & -0.709 & -0.709 & -0.709 & -0.708 & -0.709 \\
& Hy- MOM & 0.335 & 0.435 & 0.67 & 0.721 & 0.76 \\
\hline & & & & & & \\
\hline
\end{tabular}

Table 6. RMSE amount for estimating $\sigma$ with moment and hybrid moment methods in case of unknown $\lambda, \sigma$ and $\alpha$ for the generalized Weibull distribution (10000 samples are generated with $\lambda=0.4, \alpha=0.4$ and different values of $\sigma$ )

\begin{tabular}{llccccc}
\hline \multirow{2}{*}{$\sigma$} & \multirow{2}{*}{ Method } & \multicolumn{5}{c}{ Sample size } \\
\cline { 3 - 7 } & & 5 & 15 & 50 & 100 & 200 \\
\hline 0.1 & MOM & 41.504 & 23.994 & 13.147 & 9.297 & 6.574 \\
& Hy- MOM & 41.504 & 23.994 & 13.147 & 9.297 & 6.574 \\
\hline 0.5 & MOM & 25.568 & 14.824 & 8.129 & 5.751 & 4.066 \\
& Hy- MOM & 25.568 & 14.824 & 8.129 & 5.751 & 4.006 \\
\hline 0.8 & MOM & 13.557 & 7.88 & 4.325 & 3.061 & 2.164 \\
& Hy- MOM & 13.568 & 7.904 & 4.398 & 3.163 & 2.286 \\
\hline 1 & MOM & 5.799 & 3.315 & 1.812 & 1.284 & 0.907 \\
& Hy- MOM & 11.491 & 8.253 & 5.182 & 3.85 & 2.823 \\
\hline 1.2 & MOM & 3.278 & 1.433 & 0.706 & 0.486 & 0.341 \\
& Hy- MOM & 13.089 & 9.607 & 6.114 & 4.563 & 3.356 \\
\hline 1.4 & MOM & 10.474 & 5.795 & 3.137 & 2.21 & 1.563 \\
& Hy- MOM & 14.587 & 10.893 & 7.02 & 5.261 & 3.881 \\
\hline 1.6 & MOM & 17.949 & 10.146 & 5.526 & 3.9 & 2.758 \\
& Hy- MOM & 16.029 & 12.116 & 7.897 & 5.942 & 4.395 \\
\hline 1.8 & MOM & 25.202 & 14.358 & 7.838 & 5.534 & 3.914 \\
& Hy- MOM & 17.47 & 13.28 & 8.746 & 6.604 & 4.898 \\
\hline 2 & MOM & 32.054 & 18.354 & 10.033 & 7.086 & 5.013 \\
& Hy- MOM & 18.99 & 14.388 & 9.561 & 7.245 & 5.386 \\
\hline & & & & & &
\end{tabular}


Table 7. Bias amount for estimating $\sigma$ with moment and hybrid moment methods in case of unknown $\lambda, \sigma$ and $\alpha$ for the generalized Weibull distribution (10000 samples are generated with $\lambda=0.1, \alpha=0.4$ and different values of $\sigma$ )

\begin{tabular}{llccccc}
\hline \multirow{2}{*}{$\sigma$} & \multirow{2}{*}{ Method } & \multicolumn{5}{c}{ Sample size } \\
\cline { 2 - 6 } & & 5 & 15 & 50 & 100 & 200 \\
\hline 0.1 & MOM & 0.965 & 0.969 & 0.971 & 0.971 & 0.971 \\
& Hy- MOM & 0.965 & 0.969 & 0.971 & 0.971 & 0.971 \\
\hline 0.5 & MOM & 0.62 & 0.627 & 0.629 & 0.63 & 0.63 \\
& Hy- MOM & 0.62 & 0.627 & 0.629 & 0.63 & 0.63 \\
\hline 0.8 & MOM & 0.355 & 0.364 & 0.366 & 0.367 & 0.367 \\
& Hy- MOM & 0.418 & 0.52 & 0.624 & 0.676 & 0.72 \\
\hline 1 & MOM & 0.18 & 0.189 & 0.192 & 0.192 & 0.193 \\
& Hy- MOM & 0.47 & 0.621 & 0.757 & 0.824 & 0.881 \\
\hline 1.2 & MOM & 0.007 & 0.017 & 0.02 & 0.02 & 0.02 \\
& Hy- MOM & 0.526 & 0.715 & 0.884 & 0.967 & 1.038 \\
\hline 1.4 & MOM & -0.162 & -0.153 & -0.15 & -0.15 & -0.15 \\
& Hy- MOM & 0.574 & 0.803 & 1.006 & 1.106 & 1.19 \\
\hline 1.6 & MOM & -0.327 & -0.318 & -0.316 & -0.316 & -0.316 \\
& Hy- MOM & 0.617 & 0.885 & 1.122 & 1.239 & 1.337 \\
\hline 1.8 & MOM & -0.483 & -0.478 & -0.476 & -0.476 & -0.476 \\
& Hy- MOM & 0.657 & 0.961 & 1.233 & 1.367 & 1.48 \\
\hline 2 & MOM & -0.632 & -0.629 & -0.628 & -0.629 & -0.629 \\
& Hy- MOM & 0.688 & 1.032 & 1.339 & 1.49 & 1.617 \\
\hline & & & & & & \\
\hline
\end{tabular}

Table 8. RMSE amount for estimating $\sigma$ with moment and hybrid moment methods in case of unknown $\lambda, \sigma$ and $\alpha$ for the generalized Weibull distribution (10000 samples are generated with $\lambda=0.1, \alpha=0.4$ and different values of $\sigma$ )

\begin{tabular}{llccccc}
\hline \multirow{2}{*}{$\sigma$} & \multirow{2}{*}{ Method } & \multicolumn{5}{c}{ Sample size } \\
\cline { 3 - 7 } & & 5 & 15 & 50 & 100 & 200 \\
\hline 0.1 & MOM & 43.162 & 25.031 & 13.73 & 9.711 & 6.867 \\
& Hy- MOM & 43.162 & 25.031 & 13.73 & 9.711 & 6.867 \\
\hline 0.5 & MOM & 27.809 & 16.209 & 8.904 & 6.299 & 4.455 \\
& Hy- MOM & 27.809 & 16.209 & 8.904 & 6.299 & 4.455 \\
\hline 0.8 & MOM & 16.155 & 9.44 & 5.189 & 3.671 & 2.596 \\
& Hy- MOM & 19.439 & 13.726 & 8.914 & 6.805 & 5.114 \\
\hline 1 & MOM & 8.735 & 5 & 2.734 & 1.931 & 1.364 \\
& Hy- MOM & 22.49 & 16.456 & 10.826 & 8.305 & 6.264 \\
\hline 1.2 & MOM & 3.852 & 1.258 & 0.44 & 0.262 & 0.166 \\
& Hy- MOM & 25.618 & 19.029 & 12.662 & 9.756 & 7.382 \\
\hline 1.4 & MOM & 8.462 & 4.163 & 2.158 & 1.511 & 1.064 \\
& Hy- MOM & 28.547 & 21.459 & 14.426 & 11.161 & 8.469 \\
\hline 1.6 & MOM & 15.445 & 8.355 & 4.489 & 3.165 & 2.236 \\
& Hy- MOM & 31.34 & 23.754 & 16.12 & 12.518 & 9.525 \\
\hline 1.8 & MOM & 22.343 & 12.459 & 6.752 & 4.768 & 3.371 \\
& Hy- MOM & 34.2 & 25.923 & 17.74 & 13.824 & 10.545 \\
\hline 2 & MOM & 29.061 & 16.359 & 8.902 & 6.292 & 4.449 \\
& Hy- MOM & 36.851 & 27.97 & 19.281 & 15.074 & 11.526 \\
\hline
\end{tabular}


Table 9. Bias amount for estimating $\sigma$ with moment and hybrid moment methods in case of unknown $\lambda, \sigma$ and $\alpha$ for the generalized Weibull distribution (10000 samples are generated with $\lambda=0.1, \alpha=0.2$ and different values of $\sigma$ )

\begin{tabular}{llccccc}
\hline \multirow{2}{*}{ Method } & \multicolumn{5}{c}{ Sample size } \\
\cline { 3 - 7 } & & 5 & 15 & 50 & 100 & 200 \\
\hline 0.1 & MOM & 0.926 & 0.927 & 0.928 & 0.928 & 0.928 \\
& Hy- MOM & 0.9260 .542 & 0.927 & 0.928 & 0.928 & 0.928 \\
\hline 0.5 & MOM & 0.542 & 0.544 & 0.545 & 0.545 & 0.545 \\
& Hy- MOM & 0.251 & 0.544 & 0.545 & 0.545 & 0.545 \\
\hline 0.8 & MOM & 0.252 & 0.253 & 0.254 & 0.254 & 0.254 \\
& Hy- MOM & 0.056 & 0.263 & 0.287 & 0.303 & 0.317 \\
\hline 1 & MOM & 0.256 & 0.059 & 0.06 & 0.06 & 0.06 \\
& Hy- MOM & 0.254 & 0.306 & 0.35 & 0.372 & 0.391 \\
\hline 1.2 & MOM & -0.139 & -0.136 & -0.135 & -0.135 & -0.135 \\
& Hy- MOM & 0.292 & 0.357 & 0.412 & 0.44 & 0.463 \\
\hline 1.4 & MOM & -0.334 & -0.331 & -0.33 & -0.33 & -0.329 \\
& Hy- MOM & 0.328 & 0.406 & 0.473 & 0.506 & 0.535 \\
\hline 1.6 & MOM & -0.529 & -0.525 & -0.524 & -0.524 & -0.524 \\
& Hy- MOM & 0.361 & 0.454 & 0.533 & 0.572 & 0.605 \\
\hline 1.8 & MOM & -0.723 & -0.72 & -0.719 & -0.719 & -0.719 \\
& Hy- MOM & 0.391 & 0.5 & 0.592 & 0.636 & 0.675 \\
\hline 2 & MOM & -0.918 & -0.915 & -0.913 & -0.913 & -0.913 \\
& Hy- MOM & 0.42 & 0.545 & 0.649 & 0.7 & 0.743 \\
\hline & & & & & & \\
\hline
\end{tabular}

Table 10. RMSE amount for estimating $\sigma$ with moment and hybrid moment methods in case of unknown $\lambda, \sigma$ and $\alpha$ for the generalized Weibull distribution (10000 samples are generated with $\lambda=0.1, \alpha=0.2$ and different values of $\sigma$ )

\begin{tabular}{llccccc}
\hline \multirow{2}{*}{$\sigma$} & Method & \multicolumn{5}{c}{ Sample size } \\
\cline { 3 - 7 } & & 5 & 15 & 50 & 100 & 200 \\
\hline 0.1 & MOM & 41.4 & 23.942 & 13.122 & 9.279 & 6.562 \\
& Hy- MOM & 41.4 & 23.942 & 13.122 & 9.279 & 6.562 \\
\hline 0.5 & MOM & 24.25 & 14.056 & 7.709 & 5.453 & 3.857 \\
& Hy- MOM & 24.25 & 14.056 & 7.709 & 5.453 & 3.857 \\
\hline 0.8 & MOM & 11.243 & 6.544 & 3.595 & 2.543 & 1.799 \\
& Hy- MOM & 11.243 & 6.834 & 4.073 & 3.037 & 2.249 \\
\hline 1 & MOM & 2.692 & 1.549 & 0.849 & 0.6 & 0.425 \\
& Hy- MOM & 11.686 & 7.987 & 4.979 & 3.733 & 2.772 \\
\hline 1.2 & MOM & 6.31 & 3.528 & 1.912 & 1.349 & 0.952 \\
& Hy- MOM & 13.503 & 9.346 & 5.871 & 4.415 & 3.286 \\
\hline 1.4 & MOM & 14.977 & 8.547 & 4.663 & 3.296 & 2.329 \\
& Hy- MOM & 15.221 & 10.66 & 6.745 & 5.086 & 3.793 \\
\hline 1.6 & MOM & 23.677 & 13.572 & 7.416 & 5.242 & 3.705 \\
& Hy- MOM & 16.853 & 11.933 & 7.601 & 5.746 & 4.293 \\
\hline 1.8 & MOM & 32.381 & 18.597 & 10.168 & 7.188 & 5.081 \\
& Hy- MOM & 18.407 & 13.17 & 8.442 & 6.397 & 4.788 \\
\hline 2 & MOM & 41.081 & 23.618 & 12.917 & 9.132 & 6.456 \\
& Hy- MOM & 19.892 & 14.374 & 9.269 & 7.039 & 5.277 \\
\hline & & & & & &
\end{tabular}

\section{Copyrights}

Copyright for this article is retained by the author(s), with first publication rights granted to the journal.

This is an open-access article distributed under the terms and conditions of the Creative Commons Attribution license (http://creativecommons.org/licenses/by/3.0/). 